\title{
Desafíos para la enfermería de salud mental después del COVID-19
}

\author{
Challenges for mental health nursing after COVID-19 \\ Desafios para a enfermagem em saúde mental após o COVID-19
}

Daniel Martinez-Esquivel ${ }^{1}$

\section{Resumen}

La enfermería de salud mental se centra en el cuidado a partir de las relaciones interpersonales, es guiada por un marco teórico y metodológico propio que la convierten en una profesión autónoma. La pandemia por COVID-19 ha afectado la salud mental de muchas personas poniendo a prueba todos los niveles de atención de la salud. Por lo que en este artículo se busca describir las realidades, los avances y los desafíos para la enfermería de salud mental después de la pandemia por COVID-19. Se exponen algunos elementos que podrían mejorar la calidad de los servicios en esta área, dentro de los que se mencionan el desarrollo de: Investigación en e-salud, enfermería de salud mental en el hospital general, cuidado remoto, manejo del duelo, medios de comunicación y cooperación en equipo. La enfermería de salud mental debe aprovechar los desafíos que genera la pandemia por COVID-19 para innovar su ejercicio profesional basado en evidencia científica.

Palabras clave: Coronavirus; Enfermería; Salud Mental; Atencion de enfermeria

\section{Abstract}

Mental health nursing focuses on care from interpersonal relationships, is guided by its theoretical framework and methodological framework that make it an autonomous profession. The COVID-19 pandemic has affected the mental health of many people, testing all levels of health care. Therefore, this article seeks to describe the realities, advances, and challenges for mental health nursing after the COVID-19 pandemic. Some elements that could improve the quality of the services are exposed, within which the development of: e-health research, mental health nursing in the general hospital, remote care, grief management, media and cooperation are men-

\author{
Autor de correspondencia* \\ 1* Máster en enfermería de salud mental, \\ docente en la Escuela de Enfermería. San \\ José, profesor adjunto en la Universidad de \\ Costa Rica. Correo: daniel.martinezesqui- \\ vel@ucr.ac.cr in
}

(C) Universidad Francisco de Paula Santander. Este es un artículo bajo la licencia CC-BY-NC-ND (c) $(i) \Theta(\Theta$

Recibido: 13 marzo 2020. Aprobado: 16 julio 2020.

Para citar este artículo/ To reference this article/ Para citar este artigo/

Martinez-Esquivel D. Desafíos para la enfermería de salud mental después del COVID-19. Rev. cienc. cuidad. 2020;

17(3):122-129. https://doi.org/10.22463/17949831.2413 
tioned. team up. Mental health nursing must take advantage of the challenges generated by the COVID-19 pandemic to innovate their professional practice based on scientific evidence.

Key words: Coronavirus; Nursing; Mental Health.

ISSN-PRINT

$1794-9831$

\section{Resumo}

A enfermagem em saúde mental concentra-se no cuidado a partir das relações interpessoais, é guiada por seu próprio referencial teórico e metodológico que o torna uma profissão autônoma. A pandemia do COVID-19 afetou a saúde mental de muitas pessoas, testando todos os níveis de assistência médica. Portanto, este artigo busca descrever as realidades, avanços e desafios da enfermagem em saúde mental após a pandemia COVID-19. São expostos alguns elementos que podem melhorar a qualidade dos serviços, nos quais são mencionados o desenvolvimento de: pesquisa em e-saúde, enfermagem em saúde mental no hospital geral, cuidados remotos, gerenciamento de luto, mídia e cooperação. em equipe. A enfermagem em saúde mental deve aproveitar os desafios gerados pela pandemia do COVID-19 para inovar sua prática profissional com base em evidências científicas.

Palavras chave: Coronavirus; Enfermagem; Saúde Mental.

\section{Introducción}

El presente artículo es una reflexión sobre los desafíos a los que se enfrenta la enfermería de salud mental ante una circunstancia disruptiva como la pandemia por COVID-19, los cuáles podrían representar una oportunidad para que esta disciplina se (re)posicione como un recurso de calidad que responde a las necesidades de cuidado de las personas en el mundo.

En este sentido, la enfermería de salud mental es en sí misma un proceso terapéutico que se desarrolla en las relaciones interpersonales de cuidado y de ayuda con el otro. Las relaciones interpersonales, se basan en la interacción de la enfermera con la persona para satisfacer sus necesidades a través del crecimiento cooperativo. Su práctica profesional se fundamenta en el autoconocimiento, que parte del hecho de conocerse para tener la capacidad de conocer a otros; la empatía haciendo referencia a ponerse en el lugar del otro y la compasión a partir de la cual se comprende al otro, como herramientas que le permiten conec- tar de manera humanizada con los pensamientos, los sentimientos y las conductas que influyen en la calidad de vida relacionada a la salud de individuos, familias o comunidades $(1,2)$.

Los cuidados de enfermería de salud mental están presentes en los diferentes niveles de atención de los sistemas de salud (3). Implementa estrategias de promoción, prevención, recuperación o rehabilitación. Se fundamenta en teorías o modelos como las relaciones interpersonales en enfermería, los sistemas de Neuman, la recuperación de la marea en la salud mental, el cuidado transpersonal, entre otros (4). Además, su eje metodológico se basa en las cinco etapas del proceso de enfermería (valoración, diagnóstico, planeación, ejecución y evaluación) sustentado en el lenguaje taxonómico de enfermería (5).

También, participa en la construcción de conocimiento científico y de evidencia empírica por medio de la investigación que actualiza y sustenta su ser, saber y hacer. La enfermería de salud mental es responsable al brindar cuidados eficientes, efectivos y de calidad acorde a las dinámicas contemporáneas (6). Sin embargo, las dinámicas son 
flexibles y cambiantes, por ejemplo, la afectación a nivel global por la pandemia por COVID-19; las cuales están siendo impactadas por el distanciamiento social, la restricción a lugares públicos y privados, el desempleo, entre otras, que han generado sufrimiento psíquico $(7,8)$.

El COVID-19 cambió la vida cotidiana de todas las personas. Su expansión acelerada provocó que en cuestión de semanas fuera declarado pandemia (9), haciendo que sea el foco de atención de muchas personas académicas y científicas que han buscado comprender este fenómeno por medio del desarrollo de investigaciones, de revisiones o de reflexiones. Debido a la actualidad de los hechos, la producción del conocimiento es "en vivo" por lo que adoptar o contradecir una posición sería algo prematuro.

Ante esto, la enfermería de salud mental debe fortalecer su rol de liderazgo y vanguardista en la salud, por lo que en este artículo se busca responder al siguiente objetivo: Describir las realidades, los avances y los desafíos para la enfermería de salud mental después de la pandemia por COVID-19.

\section{Desarrollo}

La pandemia por COVID-19 ha afectado la salud mental de muchas personas alrededor del mundo. Desde el inicio de la emergencia sanitaria, los gobiernos fueron adoptando medidas cada vez más drásticas para contener la transmisión del virus, tomando como estrategia común el distanciamiento social. A pesar de que el distanciamiento social se ha considerado como una de las mejores formas de combatir el COVID-19, su establecimiento provocó en algunos sectores descontento, enojo y frustración, pero también aburrimiento (7).

A causa del distanciamiento social aumentó el alejamiento de las personas con sus redes de apoyo. Disminuyó el contacto entre familiares y amigos, las dinámicas en los espacios de recreación o la práctica del deporte, debido a una serie de lineamientos sanitarios que restringen el acceso a lugares públicos como los parques o a lugares privados como los centros comerciales e incluso los hogares de las personas con las que no se convive, desatando una crisis (8) acompañada de estrategias de afrontamiento des adaptativas como el sedentarismo, el consumo de drogas, las dietas altas en grasa, entre otras. En este sentido, el estancamiento cotidiano se encargó de provocar diversas alteraciones de salud mental.
Dichas alteraciones de la salud mental incluyen aumento del estrés, de la ansiedad, del temor o del pánico, de la depresión, de la psicosis e inclusive del suicidio $(8,10,11)$. Menciona Cao et al (12), que estos trastornos han afectado a todos los grupos etarios, desde la niñez hasta la adultez mayor. Esta situación debe ser valorada para que sean tomadas las medidas necesarias de acompañamiento y así evitar su cronicidad, ya que la desatención de la salud mental genera sociedades más tristes.

En Costa Rica, el distanciamiento social ha favorecido la contención del COVID-19 evitando la saturación de los servicios de salud. Al día de hoy, 26 de mayo de 2020, se contabilizan 956 casos de los cuales hay 634 recuperados y diez fallecidos (13). El país tiene datos muy favorables respecto al resto de América. Sin embargo, en otros países no se tuvo el mismo efecto como el caso de Estados Unidos, Brasil o Reino Unido, quienes presentan datos al 26 de mayo de 2020 de 1.723 .999 casos (100.497 fallecidos), 377.780 casos (23.622 fallecidos), y 265.227 casos (37.048 fallecidos) respectivamente (14).

En consecuencia, el panorama muestra miles de personas fallecidas que no tuvieron la oportunidad de despedirse de sus seres queridos, muriendo en condiciones solitarias o acompañadas de personal sanitario. Esto ha provocado afectación de la salud mental en dos líneas. En primer lugar, muchas familias están viviendo un proceso de duelo o incluso de duelo complicado debido a los lineamientos generales para la vigilancia de la infección por coronavirus, como el aislamiento de las personas hospitalizadas o la entrega de cadáveres en una bolsa sellada (13).

Y, en segundo lugar, el personal sanitario que se encuentra en la línea directa de atención de las personas hospitalizadas por COVID-19, se enfrenta al sufrimiento de usuarios y familiares, así como a la muerte. Su exposición constante provoca cansancio del rol, debido a la exigencia de largas jornadas de trabajo, temor de contagiarse y contagiar a otros, asi como al estrés posterior a este evento traumático (15).

El COVID-19 demostró, en cierta medida, la fragilidad de la salud mental global. Ha sido el detonante que reflejó el estrés y el sufrimiento que la vida cotidiana contemporánea genera en muchas personas ante las demandas sociales y económicas. También, ha permitido la reflexión y la recuperación de algunos valores de la vida realmente importantes como son la solidaridad, la cercanía con los seres queridos o el sentimiento de sentirse libre. Ante la postura de que el mundo nunca va a 
ser igual después del COVID-19, se asume que la enfermería de salud mental tampoco debería de serlo. A continuación, algunos desafíos:

\section{Desarrollo de la investigación.}

La investigación es el medio por el cual la enfermería de salud mental puede crear conocimientos nuevos y respaldar las actividades de cuidado que realiza. La premisa de formación a lo largo de la vida estimula la preparación científica continua y garantiza los servicios de cuidado adecuados. En este sentido, la investigación debe estar integrada en el hacer de la profesión, provocando cuestionamientos, comparaciones y validaciones de sus teorías, instrumentos o intervenciones. De esta manera se convierte en el único motor de crecimiento y desarrollo (6).

Sumado a esto, una "revolución" permitiría adaptarse a la era digital siendo necesario el desarrollo de investigaciones, como por ejemplo, las intervenciones e-salud. Estas favorecerían la creación y el desarrollo de programas de tratamiento durante la crisis, el proceso agudo, la etapa de mantenimiento o la promoción de la salud ante circunstancias de sufrimiento o trastorno mental. Las investigaciones e-salud se enfocan en el cuidado que se provee a través de internet y de la tecnología para mejorar la salud. Siguen un proceso riguroso en el que se toma en cuenta la evidencia existente cuando está disponible, se vinculan las partes interesadas en su planteamiento y desarrollo, se valora su viabilidad y factibilidad, y se evalúa la eficiencia y eficacia de su implementación. En países como Noruega, se han desarrollado experiencias centradas en las intervenciones de autogestión sobre enfermedades crónicas, obteniendo soluciones innovadoras para personas usuarias, personal de enfermería y otros profesionales de la salud para el manejo del dolor o del estrés (16).

En Costa Rica, ya se han impulsado estas iniciativas formalmente. La última se desarrolló a principios del año 2020 con el curso "Intervenciones innovadoras e-Health en la Investigación de enfermería: un enfoque desde los diseños mixtos de investigación, desarrollo, evaluación e implementación", que contó con enfermeras de distintas áreas como docentes de la Escuela de Enfermería de la Universidad de Costa Rica, personal de la Caja Costarricense de Seguro Social, representantes del Colegio de Enfermeras de Costa Rica, entre otros. Cabe mencionar que en los grupos de trabajo hubo uno de enfermería de salud mental (17). Este curso incluyó la formu- lación de diferentes propuestas dentro de las que destaca "Balance", una propuesta de una intervención e-salud para el autocuidado de la salud mental en estudiantes universitarios.

\section{Enfermería de salud mental en el hospital ge- neral.}

Las experiencias relacionadas a la enfermedad física se convierten en procesos que generan estrés. Sin el debido acompañamiento, el estrés puede transformarse en ansiedad, depresión o duelo complicado (5), provocando de esta manera dificultades en la recuperación de la salud. La pandemia por el coronavirus ha sido reflejo de cómo una enfermedad física trasciende el área biológica para afectar el área mental, sumando manifestaciones mentales a la sintomatología común del COVID-19. Además, no solo las personas usuarias pueden verse afectadas sino que el personal de salud, al verse sometido a una carga mental de trabajo patológica debido a la sobresaturación de los servicios de salud.

Algunos de los ejemplos que justifican lo anterior son los casos de Pakistán o de Wuhan. Por un lado, en Pakistán, se ha registrado un aumento de los casos de crisis relacionados con estrés, desesperanza, odio y miedo a causa de la expansión del coronavirus. Las autoridades de este país promueven en la población la adopción de conductas saludables que les permita afrontar las adversidades de este brote, como son: Estrategias para el desarrollo personal y construcción de la resiliencia, actividad física y una dieta balanceada. Igualmente, los profesionales de la salud mental brindan asesorías basadas en la evidencia empírica, desmitificando la información falsa con el objetivo de disminuir los problemas de salud mental. El gobierno valora la inclusión de intervenciones de salud mental y de crisis para mitigar de manera oportuna el impacto de esta pandemia (18).

Por otro lado, Kang et al. (19) evidenciaron que alrededor del $30 \%$ de las enfermeras y de los médicos en Wuhan, sufrieron algún tipo de trastorno mental moderado o severo, accediendo el $50 \%$ a diferentes recursos de salud mental. Asimismo, el $36.3 \%$ recurrió a materiales de salud mental como libros; el $50.4 \%$ utilizó métodos de autoayuda disponibles en medios de comunicación, y el $17.5 \%$ tuvo la oportunidad de una intervención individual. No obstante, los servicios de salud mental son limitados y el apoyo para los trabajadores de primera línea es escaso. 
ISSN-PRINT

1794-9831

E-ISSN 2322-7028

Vol. 17 No. 3

Sep - Dic 2020

Cúcuta, Colombia
Para prevenir cualquier complicación psicológica o psiquiátrica, se hace necesario invertir en los servicios de salud mental que apoyen a las enfermeras, a los médicos generalistas y a los profesionales de otras especialidades en el cuidado de las personas que sufren enfermedades agudas o crónicas en los servicios de medicinas y cirugías, pero también en el cuidado de los mismos profesionales.

Así como la psiquiatría de enlace se encarga del diagnóstico y del tratamiento médico de los trastornos mentales en un hospital general (20), la enfermería de salud mental se enfoca en las relaciones de cuidado, en la cual pueden desempeñarse diferentes roles como el de enseñanza o el de asesoramiento (2) para el acompañamiento dimensionado desde la promoción y la prevención. Conjuntamente, es interlocutora entre la persona enferma, la familia y los profesionales sanitarios, promotora del cuidado humanizado, consultora de cuidados especializados, referente para programas específicos y fomento de ambientes terapéuticos en los equipos de trabajo(3).

\section{Cuidado remoto.}

A pesar de que en muchos servicios de salud alrededor del mundo se ha utilizado la tele-enfermería como medio para garantizar el acceso, no se ha considerado como una estrategia de primera línea. No obstante, la pandemia por COVID-19 ha relanzado el uso de las tecnologías de infor- mación y comunicación (TIC's) como forma de manejo para brindar cuidado (10).

Algunos ejemplos son, la región de Lombardía donde están implementando servicios remotos como prioridad para garantizar los servicios en todos los niveles y el seguimiento de aquellas personas que sufren de depresión, ansiedad, psicosis o conductas suicidas (4). También, es el caso de Australia (21) donde se han implementado consultas remotas por llamada telefónica. Estas consultas se realizan por un equipo integrado de enfermeras, psiquiatras, psicólogos y trabajadoras sociales. De momento, constatan que las llamadas han sido bien recibidas por los usuarios.

En Costa Rica, surge el proyecto Míshka en el contexto de la emergencia nacional por COVID-19, el cual consiste en una coalición de profesionales de la salud mental (enfermeras de salud mental y psicólogos) que tiene como propósito la atención durante la crisis de personas universitarias a través del uso de la video llamada. El proyecto se basa en la técnica de intervención en crisis y en la referencia de casos (22). Es importante mencionar que si bien las enfermeras de salud mental como los psicólogos basan su acompañamiento en esta técnica, cada profesional utiliza elementos propios de su disciplina. Para el caso de la enfermería de salud mental, el uso de teorías y modelos de enfermería y del lenguaje taxonómico (5). En relación a este país, algunas de las iniciativas que se han desarrollado en el contexto de la pandemia se presentan en la tabla 1.

Tabla 1. Algunas iniciativas para el abordaje de la salud mental en Costa Rica dentro del contexto de la pandemia por COVID-19.

\begin{tabular}{|c|c|c|c|c|}
\hline Nombre & Actividad & Población meta & $\begin{array}{l}\text { Profesionales parti- } \\
\text { cipantes }\end{array}$ & Responsable \\
\hline Proyecto Míshka & $\begin{array}{l}\text { Intervención en caso } \\
\text { de crisis a través de } \\
\text { videollamada. } \\
\text { Grupos de apoyo. }\end{array}$ & $\begin{array}{l}\text { Comunidad universi- } \\
\text { taria. }\end{array}$ & $\begin{array}{l}\text { Enfermeros(as) de } \\
\text { salud mental y psicó- } \\
\text { logos(as). }\end{array}$ & $\begin{array}{l}\text { Oficina de Bienestar y } \\
\text { Salud de la Universidad de } \\
\text { Costa Rica. }\end{array}$ \\
\hline $\begin{array}{l}\text { Servicio de crisis } \\
\text { en salud mental. }\end{array}$ & $\begin{array}{l}\text { Línea para llamadas } \\
\text { telefónicas. }\end{array}$ & $\begin{array}{l}\text { Enfermeras y enfer- } \\
\text { meros. }\end{array}$ & $\begin{array}{l}\text { Enfermeros(as) de } \\
\text { salud mental. }\end{array}$ & $\begin{array}{l}\text { Colegio de enfermeras de } \\
\text { Costa Rica. }\end{array}$ \\
\hline $\begin{array}{l}\text { Juntxs nos pode- } \\
\text { mos cuidar }\end{array}$ & $\begin{array}{l}\text { Línea para llamadas } \\
\text { telefónicas. } \\
\text { Cápsulas informa- } \\
\text { tivas. } \\
\text { Facebook live. } \\
\text { Videos. }\end{array}$ & Población general. & $\begin{array}{l}\text { Psicólogos(as). } \\
\text { Profesionales de } \\
\text { disciplinas afines. }\end{array}$ & $\begin{array}{l}\text { Colegio de profesionales } \\
\text { en psicología de Costa } \\
\text { Rica. } \\
\text { Ministerio de Salud de } \\
\text { Costa Rica. } \\
\text { Equipo de seguridad de } \\
\text { Facebook e Instagram. }\end{array}$ \\
\hline
\end{tabular}

Fuente: Oficina de Bienestar y Salud, Universidad de Costa Rica. Colegio de enfermeras de Costa Rica. Colegio de profesionales en psicología de Costa Rica. 
Sin embargo, a pesar de estas iniciativas, el reto surge en que los profesionales de la salud mental deben (re)entender las nuevas formas de interacción humana para ejercer su profesión, "virtualizando" el acto terapéutico de la comunicación facilitadora, la escucha activa y la empatía.

\section{Manejo del duelo.}

En el mundo se registran 350.612 fallecidos por COVID-19 al 26 de mayo de 2020, lo cual significa una cantidad considerable de muertes por una misma causa en un periodo corto de tiempo (sumado a las muertes por otras causas que no se detienen). Esto ha provocado un desbordamiento de la capacidad de los servicios de salud. La relación de alta mortalidad que se genera con el COVID-19, ha provocado desajustes en la salud mental de los profesionales sanitarios y las familias (24).

Se ha evidenciado estrés postraumático en el personal sanitario y se proyecta que un porcentaje sufra de síndrome de burnout, debido a enfrentarse con la muerte cara a cara, entre otras cosas. También se data duelo complicado en las familias debido a las normas de aislamiento. Y una faltante de personal calificado para brindar acompañamiento $(15,23)$.

La enfermería de salud mental debe tomar un papel protagónico en el desarrollo de programas de entrenamiento y acompañamiento sobre cuidados al final de la vida y de la muerte, enfocados en la implementación del apoyo educativo, del manejo del estrés, del mindfulness, de la meditación o del empoderamiento, mediante estrategias como las llamadas telefónicas, las video llamadas o el 'chat' $(8,10,15)$.

\section{Medios de comunicación.}

La enfermería de salud mental debe aprovechar la era digital para generar recursos accesibles para toda la población. Estos recursos deben basarse en la mejor evidencia científica posible para garantizar resultados favorables en su aplicación y presentarse en idiomas comprensibles para cada región. Además, deben aprovecharse los diferentes medios de comunicación como los sitios web, los webinar, web feed, podcast, blog, mensajería instantánea, redes sociales y foros de internet; de manera que quien tenga acceso, se identifique y se vincule de manera tal que adopte pensamientos positivos y conductas saludables (8).

\section{Cooperación en equipo.}

El desarrollo de equipos de salud se viene tejiendo en los sistemas de salud, siendo reimpulsados por la estrategia de atención primaria de salud. El reto se dimensiona en que continúen conformándose como equipos acordes a la realidad social desde la cooperación, el apoyo mutuo y los conocimientos compartidos sin que exista la competencia individual o luchas de poder, sino que la dirección sea el bienestar común (24).

La enfermería de salud mental podría posicionarse a partir de su rol de liderazgo como promotora de la conducta prosocial en los servicios de salud. La conducta prosocial, se refiere a todos los comportamientos que tengan como fin el bien común por encima del bien individual (25), como por ejemplo el distanciamiento social o el acceso universal a la salud. El hecho de que existan equipos prosociales enfocaría la mirada del sistema de salud en todas las poblaciones y grupos etarios, evitando que la exclusión social limite la atención de las personas con enfermedad mental.

Lo expuesto anteriormente son solo algunos desafíos propuestos por el autor en base a su perspectiva y a la evidencia generada durante la pandemia por COVID-19. Como menciona Yao (26), se debe analizar cada uno en función de las oportunidades sociales y económicas de quienes accedan a los servicios de salud mental, sus posibilidades en el manejo de las TIC's y la evaluación de su efectividad en el mejoramiento de la calidad de vida de las personas.

\section{Conclusiones.}

- La pandemia por COVID-19 evidenció las necesidades latentes a nivel global sobre la salud mental agrietando la vida de muchas personas al afrontar situaciones de estrés, ansiedad, depresión o duelo. Debido a esto, se vuelve una necesidad prioritaria la inversión de recursos en salud mental que puedan satisfacerlas así como la priorización en los sistemas de salud de un plan de acción que permita atenderlas a través de programas de promoción y prevención.

- La enfermería de salud mental debe aprovechar los desafíos generados por la pandemia por COVID-19 en cuanto a la innovación del ejercicio profesional para (re)posicionarse en la comunidad científica y en la población en general, para fortalecer las prácticas de cuidado de la salud mental mediante la e-Heal- 
th, el uso de TIC's, la formación a lo largo de la vida y el trabajo interdisciplinario.

- La enfermería de salud mental debe asumir el desafío de robustecer su currículo con la investigación, el cuidado remoto y la adquisición de nuevos conocimientos relacionados a la promoción, la prevención, la recuperación, el mantenimiento y la rehabilitación de la salud mental para reafirmar que es una disciplina indispensable en todos los niveles de atención en salud.

- La enfermería de salud mental debe enfrentarse al desafío de acompañar y asesorar a la población en general, creando recursos de autogestión de la salud accesibles para todos y todas en los diferentes medios de comunicación como internet, redes sociales, radio, televisión y otras plataformas.

- La enfermería en salud mental debe aflorar su liderazgo, su cooperación, su capacidad de comunicación y de trabajo en equipo, para integrar equipos de salud que sean eficientes y eficaces en el desarrollo de políticas y programas sobre la atención de la salud mental garantizando su implementación. Al respecto, sólo a través del trabajo interdisciplinario se puede fomentar la recuperación de la salud mental en las personas que lo necesiten.

\section{Conflicto de intereses}

El autor declara no tener ningún conflicto de interes

\section{Referencias bibliográficas}

1. Suhariyanto Rr, Tutik S, Titin U. Improving the interpersonal competences of head nurses through Peplau's theoretical active learning approach. Enferm Clin. 2018; 28(1A): 149-153. DOI: https://doi. org/10.1016/S1130-8621(18)30056-1

2. Peplau H. Relaciones interpersonales en Enfermería. Un marco de referencia conceptual para la Enfermería psicodinámica. Barcelona: Salvat Editores S.A.; 1990.

3. Huizing E, Rodríguez-Gómez S y Lafuente-Robles N. La implantación de la especialidad de Enfermería de Salud Mental en Andalucía desde un enfoque comunitario. Enferm Clin. 2019; 29(6): 369-374. DOI: https://doi.org/10.1016/j.enfcli.2019.10.003

4. Zaraza Morales DR, Contreras Moreno JR. El modelo de recuperación de la salud mental y su importancia para la enfermería colombiana. Rev Colomb Psiquiat. 2018. DOI: https://doi.org/10.1016/j. rcp.2018.10.002

5. Stuart G. Principles and Practice of Psychiatric Nursing. Tenth Edition. St Louis, Missouri: Elsevier Mosby; 2013.

6. Lluch Canut MT. Enfermería de salud mental: El arte y la ciencia del cuidar. Revista española de enfermería de salud mental. 2018; 4: 2-3. DOI: http://doi.org/10.35761/reesme.2018.4.01

7. Zhu S, Wu Y, Zhu C, Hong W, Yu Z, Chen Z, Chena Z, Jiang D, Wang Y. The immediate mental health impacts of the COVID-19 pandemic among people with or without quarantine managements. Brain, Behavior, and Immunity. 2020. DOI: https://doi.org/10.1016/j.bbi.2020.04.045

8. Hiremath P, Kowshik CS, Manjunath M, Shettar M. COVID 19: Impact of lock-down on mental health and tips to overcome. Asian Journal of Psychiatry. 2020; 51 (102088). DOI: https://doi.org/10.1016/j. ajp. 2020.102088

9. Organización Mundial de la Salud. Brote de enfermerdad por coronavirus (COVID-19). 2020. Disponible en: https://www.who.int/es/emergencies/diseases/novel-coronavirus-2019?gclid=CjwKCAjw8df2BRA3EiwAvfZWaMs3KnPNqOaXxfiQDlhDYcfXeoDQ4K6qsRAru8X5-PccwhaFT2eHWBoCbmsQAvD BwE

10. Rajkumar Ravi P. "COVID-19 and mental health: A review of the existing literatura". Asian Journal of Psychiatry. 2020. DOI: https://doi.org/10.1016/j.ajp.2020.102066

11. Percudani M, Corradinb M, Moreno M, Indelicato A, Vita A. Mental Health Services in Lombardy during COVID-19 outbreak. Psychiatry Research. 2020: 288. DOI: https://doi.org/10.1016/j.psy- 
chres.2020.112980

12. Cao W, Fang Z, Hou G, Han M, Xu X, Dong J, Zheng J. The psychological impact of the COVID-19 epidemic on college students in China. Psychiatry Research. 2020; 287(112934). DOI: https://doi. org/10.1016/j.psychres.2020.112934

13. República de Costa Rica. Ministerio de Salud. Situación nacional COVID-19 [Internet]. El Ministerio; 2020 [Consultado el 25 de abril de 2020]. Disponible en: https:/www.ministeriodesalud.go.cr/index.php/centro-de-prensa/noticias/741-noticias-2020/1532-lineamientos-nacionales-para-la-vigilancia-de-la-infeccion-por-coronavirus-2019-ncov

14. Organización Mundial de la Salud. Coronavirus (COVID-19) [Internet]. Ginebra; 2020 [Consultado el 26 de mayo de 2020]. Disponible en: www.covid19.who.int

15. Vieta E, Perez V, Arango C, La psiquiatría en las secuelas de COVID-19. Revista de psiquiatría y salud mental. 2020. DOI: https://doi.org/10.1016/j.rpsm.2020.04.004

16. Børøsund E y Varsi C. Innovative eHealth interventions in nursing research. How to utilize a mixed method approach to design, development, evaluation and implementation. Ciencia y Enfermería. 2019; 25: 22. DOI: https://doi.org/10.4067/s0717-95532019000100401

17. Escuela de Enfermería, CICES. Intervenciones innovadoras e-Health en la Investigación de enfermería: un enfoque desde los diseños mixtos de investigación, desarrollo, evaluación e implementación. San José: Universidad de Costa Rica; 2020.

18. Mukhtar S. Pakistanis' Mental Health during the COVID-19. Asian Journal of Psychiatry. 2020. DOI: https://doi.org/10.1016/j.ajp.2020.102127

19. Kang L, et al. Impact on mental health and perceptions of psychological care among medical and nursing staff in Wuhan during the 2019 novel coronavirus disease outbreak: A cross-sectional study Brain, Behavior, and Immunity. 2020. DOI: https://doi.org/10.1016/j.bbi.2020.03.028

20. González M, Carreño M. Psiquiatría de enlace y medicina de enlace, nuevos alcances. Rev. Med. Clin. Condes. 2017; 28(6): 944-948. DOI: https://doi.org/10.1016/j.rmclc.2017.10.012

21. Rao Kavoora A, Chakravarthy K, Johna T. Remote consultations in the era of COVID-19 pandemic: Preliminary experience in a regional Australian public acute mental health care setting. Asian Journal of Psychiatry. 2020; 51. DOI: https://doi.org/10.1016/j.ajp.2020.102074

22. Oficina de bienestar y salud. Proyecto coalición de profesionales en salud mental de la Universidad de Costa Rica para la intervención en crisis en la comunidad universitaria dentro el contexto de la emergencia nacional por COVID-19. San José: Universidad de Costa Rica; 2020.

23. Rolim Neto M, et al. When health professionals look death in the eye: the mental health of professionals who deal daily with the 2019 coronavirus outbreak. Psychiatry Research. 2020; 288. DOI: https:// doi.org/10.1016/j.psychres.2020.112972

24. García-Rincón C. Cooperación y conducta prosocial durante el confinamiento social por COVID-19. Madrid; 2020. Disponible en: https://www.cesargarciarincon.com/2020/03/cooperacion-y-conducta-prosocial.html

25. García-Rincón C. Cómo educar las competencias y actitudes prosociales. Marcos teóricos, indicadores y actividades. Madrid: Prosocialia - Amazon Independently Published; 2019

26. Yao H, et al. Rethinking online mental health services in China during the COVID-19 epidemic. Asian Journal of Psychiatry. 2020; 50. DOI: https://doi.org/10.1016/j.ajp.2020.102015 